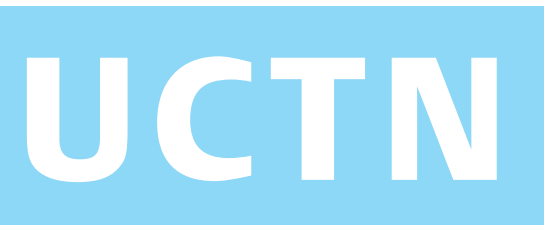

\title{
Intraductal tubular adenoma, pyloric gland-type, of the pancreas
}

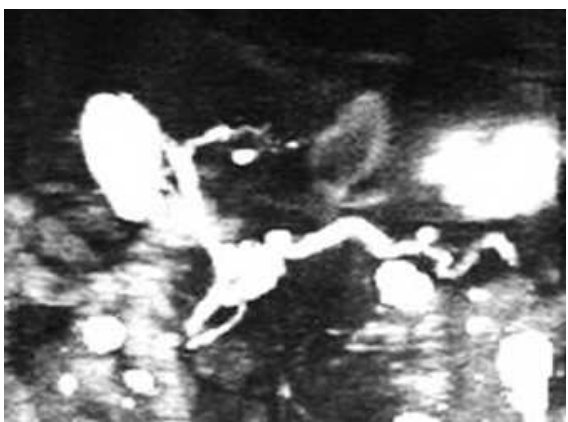

Figure 1 Magnetic resonance cholangiopancreatography demonstrated both dilatation of the main pancreatic duct and a cystic lesion in the head of the pancreas.

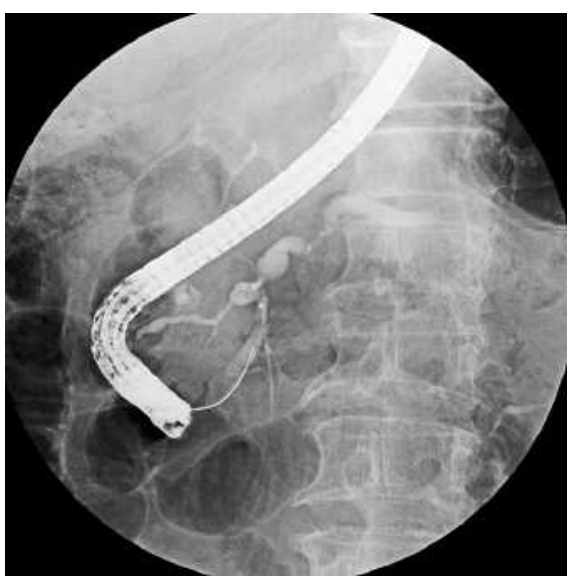

Figure 2 Endoscopic retrograde cholangiopancreatography revealed that the main duct in the pancreatic head was partially narrowed and that the distal main duct was dilated.

A 74-year-old man was admitted to our hospital for further evaluation of a pancreatic cystic lesion. Abdominal computed tomography and magnetic resonance cholangiopancreatography showed dilatation of the main pancreatic duct and a cystic lesion of the pancreatic head (Figure 1). Endoscopic retrograde cholangiopancreatography demonstrated that the main duct in the pancreatic head was partially narrowed and that the distal main duct was dilated (Figure 2 ), although the cystic lesion in the pancreatic head could not be seen. Transpapillary intraductal ultrasound clearly depicted a protruding tumor with a height of about $4.5 \mathrm{~mm}$ in the narrowed main duct (Figure 3 ). A peroral pancreatoscopic examination was then

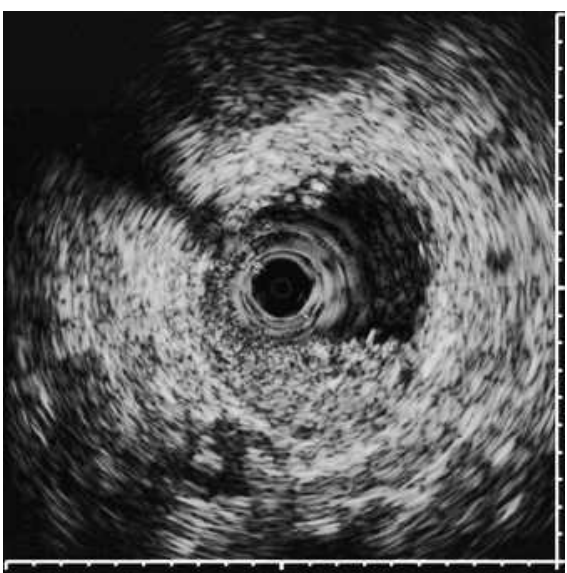

Figure 3 Transpapillary intraductal ultrasound showed the protruding tumor in the narrowed main duct.
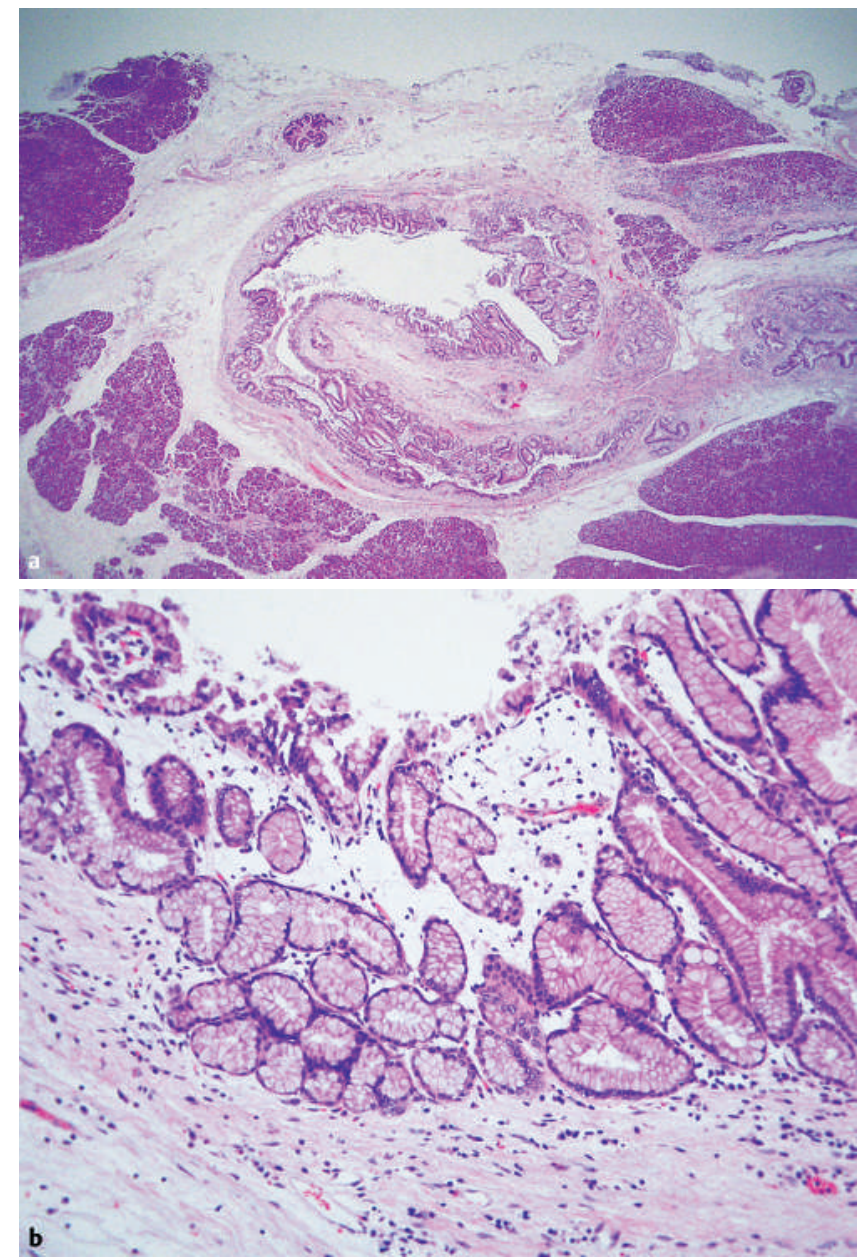

Figure 5 Histological views after staining with hematoxylin and eosin, showing a polypoid lesion protruding into the lumen of the dilated pancreatic duct (a), which was found to be composed of closely packed tubular glands (b). 
performed using a cholangioscope (CHF type BP260; Olympus Optical Co. Ltd., Tokyo, Japan). A fish-egg-like appearance was seen on peroral pancreatoscopy, with mucosal redness in the narrowed main duct (Figure 4). Cytological examination of pancreatic duct tissue showed mild cellular atypia.

Based on the preoperative diagnosis of a mixed-type intraductal papillary mucinous neoplasm, pancreatoduodenectomy was then performed. Macroscopically, the tumor in the narrowed pancreatic duct was seen to be localized, with a well-demarcated, granular, sessile appearance. Microscopically, the polypoid lesion protruding into the lumen of the dilated pancreatic duct was found to be composed of closely packed tubular glands (Figure 5). Immunohistochemical investigations showed that the tumor was completely negative for both Muc-1 glycoprotein and Muc-2 glycoprotein, but positive for Muc-6 glycoprotein and HIK1083 [1]. We therefore diagnosed this lesion as a pyloric gland-type intraductal tubular adenoma of the pancreas [2,3]. The intraductal papillary mucinous adenoma in the pancreatic head was found in the branch ducts of the pancreatic head, and was separate from the intraductal tubular adenoma of the main pancreatic duct.

Intraductal adenoma visualized by peroral pancreatoscopy has been reported only rarely in the English-language literature. In our case, pancreatoscopic and ultrasonographic findings demonstrated high protrusions and mucosal redness, both of which are regarded as signs of malignancy in intraductal papillary mucinous neoplasms [4]. These features might be important in the evaluation of intraductal ademoma in the main pancreatic duct.
H. Fukatsu', H. Kawamoto', K. Tsutsumi ${ }^{1}$, H. Kato ${ }^{1}$,

K. Hirao', N. Kurihara', T. Ogawa', E. Ishida', Y. Okamoto', H. Okada', K. Sakaguchi ${ }^{1}, \mathrm{H}$. Yanai ${ }^{2}$

${ }^{1}$ Department of Gastroenterology and Hepatology, Okayama University Graduate School of Medicine, Dentistry, and Pharmaceutical Sciences, Okayama, Japan

2 Department of Pathology, Okayama University Graduate School of Medicine, Dentistry, and Pharmaceutical Sciences, Okayama, Japan.

\section{References}

${ }^{1}$ Ishihara K, Kurihara M, Goso Y et al. Peripheral $\alpha$-linked $\mathrm{N}$-acetylglucosamine on the carbohydrate moiety of mucin derived from mammalian gastric gland mucous cells: epitope recognized by a newly characterized monoclonal antibody. Biochem J 1996; 318: 409-416

${ }^{2}$ Nakayama Y, Inoue H, Hamada Y et al. Intraductal tubular adenoma of the pancreas, pyloric gland type: a clinicopathologic and immunohistochemical study of 6 cases. Am J Surg Pathol 2005; 29: 607-616

${ }^{3}$ Albores-Saavedra J, Sheahan K, O'Riain C et al. Intraductal tubular adenoma, pyloric type, of the pancreas: additional observations on a new type of pancreatic neoplasm. Am J Surg Pathol 2004; 28: 233-238

${ }^{4}$ Hara T, Yamaguchi T, Ishihara T et al. Diagnosis and patient management of intraductal papillary-mucinous tumor of the pancreas by using peroral pancreatoscopy and intra- ductal ultrasonography. Gastroenterology 2002; 122: $34-43$

\section{Corresponding author}

\section{H. Kawamoto, MD}

Department of Gastroenterology and Hepatology

Okayama University Graduate School of Medicine, Dentistry, and Pharmaceutical Sciences

2-5-1 Shikata-cho

Okayama 700-8558

Japan

$\begin{array}{ll}\text { Fax: } & +81-86-223-5991 \\ \text { Email: } & \text { h-kawamo@ } \\ & \text { md.okayama-u.ac.jp }\end{array}$

